\title{
Protection of Geographic Data
}

\author{
Alban Gabillon and Patrick Capolsini \\ Université de la Polynésie Française, BP 6570, 98702 FAA’A, French Polynesia \\ \{Alban.Gabillon,Patrick.Capolsini\}@upf.pf
}

\begin{abstract}
A powerful and flexible authorization model should be able to cope with various security requirements. In the first part of this presentation, we define a security model to express security policies for spatial applications. Using this model we show how to express dynamic security rules that depend on various spatial contexts. A security policy specifies which objects are authorized and which objects are forbidden. However, in the framework of a geographic application displaying maps, there are several solutions for protecting a sensitive object. Sensitive objects can be hidden, masked, blurred or even replaced by fake objects. Therefore, in the second part of this presentation, we suggest a framework to specify protection mechanisms to enforce whenever a prohibition is derived from the security policy.
\end{abstract}

Keywords: Access Control, Geo-spatial Data visualization, Map service, Policy Enforcement Point.

\section{Introduction}

The core RBAC [3] authorization model considers only static security rules. However, in many applications, there is an increasing need for dynamic security rules. A dynamic security rule can be activated/deactivated depending on some context. A context can be a temporal condition, a spatial condition (like the user location), a provisional condition (like the user previous action) etc. With the growing importance of geographic information in various applications, there is a need for dynamic security rules based on some spatial contexts. In the first part of this presentation [1], we define a logic based language for writing security policies for spatial applications. In particular, we try to figure out various types of spatial contexts, and we show how to model these contexts. The first part of this presentation deals with what is represented inside the dashed rectangle of Figure 1. The security policy determines which objects are authorized and which objects are not authorized.

In the second part of this presentation [2], we define a complete framework for protecting prohibited objects. In a traditional database approach, enforcement of the security policy is simply done by removing the unauthorized objects from the final answer to the query. In a geographic database application, things are more complicated. Let us consider a map service building maps from various spatial objects. Some unauthorized objects are simply removed from the final map as it is the 
case in a traditional database application, but some other sensitive objects are protected by using methods which are specific to geographic applications. Examples of such specific methods are referred to as blurring, masking, pixelization or "cut and paste" (i.e. overlay a sensitive object with another fake objects). The second part of this presentation deals with what is inside the dotted rectangle. It breaks down as follows:

- It defines a formal framework for deriving security mechanisms to be enforced on unauthorized objects.

- It proposes a Policy Enforcement Point (PEP) algorithm to display the final map taking into account the protection mechanisms that should be enforced on unauthorized objects.

We conclude this presentation by giving a sketch of the implementation of our proposal within the framework of the OpenGIS® Styled Layer Descriptor (SLD) Profile of the OpenGIS® Web Map Service (WMS) Encoding Standard. A prototype showing the feasibility of our approach can be found at the following url:

http://pages.upf.pf/Patrick.Capolsini/rech/protect/index.htm.

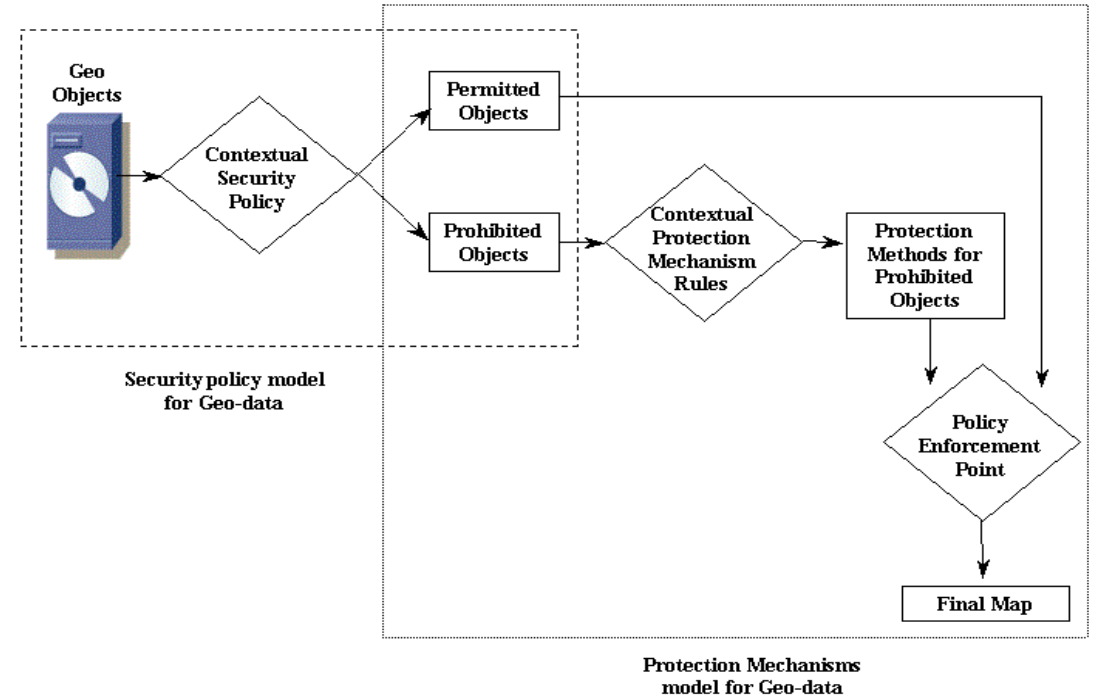

Fig. 1. Protection Mechanisms Model

\section{Acknowledgment}

This work was conducted as part of the ANR funded project under reference ANRSESUR-2007-FLUOR.

\section{References}

1. Gabillon, A., Capolsini, P.: Dynamic Security rules for Geo Data. In: Sciences, L.N.i.C. (ed.): International workshop on Autonomous and Spontaneous Security (SETOP'09), Vol. Lecture Notes in Computer Science 5939. LNCS 5939 - Springer-Verlag, St Malo, France (2009) 136-152.

2. Gabillon, A., Capolsini, P.: Rule-based Policy Enforcement Point for Map Services. ACM SIGSPATIAL GIS 2010 International Workshop on Security and Privacy in GIS and LBS (SPRINGL'10). ACM, San Jose, CA, USA (2010) 12-17 